\title{
A multilayer perceptron approach for the retrieval of vertical temperature profiles from satellite radiation data
}

\author{
Elcio H. Shiguemori, José Demisio S. da Silva, \\ Haroldo F. de Campos Velho, João C. Carvalho \\ Laboratory for Computing and Applied Mathematics - LAC \\ National Institute for Space Research - INPE \\ São José dos Campos, SP, Brazil \\ [elcio, haroldo, demisio]@lac.inpe.br
}

\begin{abstract}
In this paper a multilayer perceptron neural network is used to retrieve vertical atmospheric temperature profiles from satellite radiation data. The training set consists of data provided by the direct model characterized by the Radiative Transfer Equation (RTE) and by real radiation data from the NOAA - HIRS/2 (High Resolution Infrared Radiation) Sounder. The retrieved vertical temperature profiles are compared to radiosonde measured data. The neural network performance is compared to the results of [1] and [2] who used regularization techniques. Neural network approaches are especially advantageous due to the embed parallelism that may imply in faster vertical temperature profiles retrieving systems.
\end{abstract}

\section{1) Introduction}

The meteorological process of the atmosphere requires information of the vertical structure of the temperature and the water vapor, which are indirectly provided by satellite radiation data due to the lack of radiosonde observational stations over the globe. These pieces of information are especially important for weather analyses and data assimilation in numerical weather forecasting models in meteorology.

Satellite measured radiation is interpreted in terms of meteorological parameters, requiring the inversion of the Radiative Transfer Equation (RTE) that relates measurements of radiation at different spectral frequencies to the energy at different atmospheric regions. There is a degree of indetermination associated with the spectral resolution and the number of spectral channels.

Noises in the measuring process imply in instabilities to this solution $[3,4]$, which led to the development of different methodologies and models to improve satellite data processing. Due to the difficulties to obtain correct RTE solutions, several approaches and methods have been developed to infer information from satellite data [5-7].

In this paper a multilayer perceptron artificial neural network (ANN) is designed to solve the inversion of remotely sensed data in a multidimensional function approximation approach. The ANN retrieved temperatures are compared to those obtained [1] and [2], who used
Tikhonov and maximum entropy principle regularization techniques, respectively.

\section{2) Direct problem}

Equation (1) represents the direct problem, in which $l_{\lambda}$ is the spectral radiance, $\lambda$ is the channel frequency; $\mathfrak{I}$ is the layer to space atmospheric transmittance function; subscript $s$ denotes surface [9]; and $B$ is the Planck function which is a function of the temperature $T$ and pressure $p$ given by equation (2), where $h$ is the Planck constant, $c$ is the light speed, and $k_{B}$ is Boltzmann's constant.

$$
\begin{gathered}
I_{\lambda}(0)=B_{\lambda}\left(T_{s}\right) \mathcal{I}_{\lambda}\left(p_{s}\right)+\int_{p_{s}}^{\prime \prime} B_{\lambda_{i}}[T(p)] \frac{\partial \mathcal{S}_{\lambda}(p)}{\partial p} d p, \\
B_{\lambda}(T)=\frac{2 h c^{2} / \lambda^{5}}{e^{h c / k_{s} \lambda T}-1}
\end{gathered}
$$

The solution of equation (1) maybe approached by discretization using central finite differences (3) yielding:

$$
I_{i}=B_{i, s}\left(T_{s}\right) \mathfrak{I}_{i, s}+\sum_{j=1}^{N_{f}}\left(\frac{B_{i, j}+B_{i, j-1}}{2}\right)\left[\mathfrak{B}_{i, j}-\mathfrak{I}_{i, j-1}\right]
$$

where, $i=1, \ldots, N_{\lambda}, I_{i} \equiv I_{\lambda_{i}}(0), N_{\lambda}$ is the number of channels in the satellite, and $N_{p}$ is the number of atmospheric layers considered.

\section{3) Inverse Analysis}

Inverse problems are ill-posed problems, whose solutions do not always exist, or are not unique or stable. Following Tikhonov's approach [16], a regularized solution maybe found by choosing the appropriate temperature $T^{*}$ that minimizes the following functional

$$
J_{\alpha}[\tilde{I}, T]=\|\tilde{I}-I(T)\|_{2}^{2}+\alpha \Omega[T]
$$


where $\tilde{I}$ is the experimental data, $I(T)$ is the radiance computed from the forward model, $\Omega[T]$ denotes the regularization term given, $\alpha$ is the regularization parameter, and $\|$, is the 2-norm.

This approach maybe viewed as an optimization problem that is iteratively solved by the quasi-newtonian optimizer routine from the NAG Fortran Library [17], with variable metrics. This algorithm is designed to minimize an arbitrary smooth function subject to constraints (simple bound, linear or nonlinear constraints), using a sequential programming method. This routine has been successfully used in several previous works: in geophysics, hydrologic optics, and meteorology.

\section{4) Neural network based retrieval of vertical atmospheric temperature profiles}

This paper explores the parallelism and information distribution properties of ANNs that make them appropriate for pattern recognition, prediction and modeling, in different areas of application, such as, signal processing, image processing, financing, computer vision, engineering, etc. [8-11].

ANNs are learning systems in the sense they are trained to solve problems by exposure to exemplar patterns. The multilayer perceptron performs a mapping among input and output vectors through the connection strengths among the several neurons that are tuned during the learning process, thus changing the performance of the network.

The design of an ANN solution requires the architecture specification and the definition of training and testing sets.

In this paper Multilayer Perceptrons are trained to solve the inverse problem of inferring vertical temperature profiles from satellite radiance measured. In this application, the experimental data is simulated by adding a random perturbation to the exact solution of the direct problem, corresponding to the intrinsic error measurements in the real world, that is,

$$
\tilde{I}=I_{\text {exact }}+\sigma \mu
$$

where $\sigma$ is the standard deviation of the noise and $\mu$ is a random variable taken from a Gaussian distribution, with zero mean and unitary variance.

All numerical experiments were carried out using $5 \%$ of noise $(\sigma=0.05)$. The training set consisted of 101 profiles built from the application of Equation (5) and it is called SDB1 (Synthetic Dataset 1).

In addition, the TIGR database [15] provided 861 profiles, from which only 324 were chosen for the learning phase; 324 were chosen for the generalization phase; and 213 profiles were not considered in both phases due to existing outliers. A third training set resulted by combining both previous datasets (SBD1+TIGR).

Each input vector consists of 7 satellite radiance measurements corresponding to 7 different channels of the radiometer. The network outputs a vector with 40 values corresponding to the temperatures at 40 different heights in the atmosphere (see Figure 1).

Figure 1 shows the layers used for comparison where the error of temperature profiles were computed for each layer. This feature is important because the approach tries to achieved the best performance in layers that are of main interest for meteorological purposes (layers 1 and 2) which are below $p=100 \mathrm{hPa}$.

The testing phase, also known as the generalization test of the ANN, used 324 profiles from the TIGR data set.

The average simulation errors results at each atmospheric layer obtained with the trained MLP are shown in Tables 1, 2, and 3, for Layer-1 (20 up to $70 \mathrm{hPa}$, subdivided into 10 sublayers), Layer-2 ( 85 up to $200 \mathrm{hPa}$, subdivided into 6 sublayers), Layer-3 (250 up to $475 \mathrm{hPa}$, subdivided into 6 sublayers), and Layer-4 (500 up to 1000 $\mathrm{hPa}$, subdivided into 6 sublayers). The errors were computed by equation (6), that relates the obtained ANNs' results to radiosonde-measured data.

$$
\text { Error }=\left(\frac{1}{N} \sum_{i=1}^{N}\left(T_{i}^{\text {Radiosonde }}-T_{i}^{\text {Neural Network }}\right)^{2}\right)^{\frac{1}{2}}
$$

Where $N$ is the number of sub-layers within each layer (as defined in Figure 1). Tables 1, 2, and 3 summarize the results obtained by several different ANN architectures with different number of processing elements in one hidden layer with logistic sigmoid functions. It is to be noticed that the three tables differ in the training set used to train the network. The ANNs perform better with the combined training set.

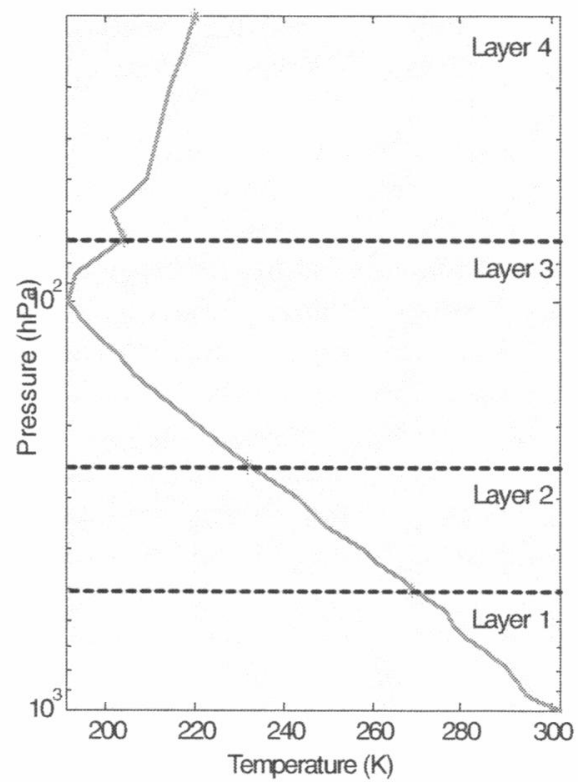

Figure 1 - Atmospheric profile - layer division. 
Table 1: Error for activation phase using SDB1.

\begin{tabular}{|c|c|c|c|c|}
\hline $\begin{array}{c}\text { Hidden } \\
\text { Neurons }\end{array}$ & Layer 4 & Layer 3 & Layer 2 & Layer 1 \\
\hline 2 & 4.027 & 2.878 & 2.364 & 1.619 \\
\hline 3 & 3.980 & 3.327 & 1.859 & 1.563 \\
\hline 4 & 3.909 & 3.456 & 2.150 & 1.545 \\
\hline 5 & 3.581 & 3.030 & 2.242 & 1.364 \\
\hline 6 & 3.271 & 2.715 & 2.126 & 1.405 \\
\hline 7 & 3.616 & 3.084 & 2.037 & 1.393 \\
\hline 8 & 3.394 & 3.199 & 1.778 & 1.372 \\
\hline 9 & 3.000 & 2.726 & 1.964 & 1.398 \\
\hline 10 & 3.363 & 3.102 & 1.854 & 1.412 \\
\hline
\end{tabular}

Table 2: Error for activation phase using TIGR.

\begin{tabular}{|c|c|c|c|c|}
\hline $\begin{array}{c}\text { Hidden } \\
\text { Neurons }\end{array}$ & Layer 4 & Layer 3 & Layer 2 & Layer 1 \\
\hline 2 & 3.203 & 2.281 & 2.616 & 3.207 \\
\hline 3 & 4.149 & 1.774 & 2.837 & 2.831 \\
\hline 4 & 5.413 & 3.250 & 2.711 & 2.498 \\
\hline 5 & 4.633 & 3.341 & 4.034 & 3.142 \\
\hline 6 & 5.110 & 3.185 & 3.838 & 1.461 \\
\hline 7 & 4.523 & 3.321 & 4.208 & 1.945 \\
\hline 8 & 4.810 & 3.047 & 4.224 & 2.198 \\
\hline 9 & 4.772 & 3.070 & 4.289 & 1.323 \\
\hline 10 & 4.264 & 2.938 & 4.500 & 1.661 \\
\hline
\end{tabular}

Table 3: Error for activation phase using SDB1+TIGR.

\begin{tabular}{|c|c|c|c|c|}
\hline $\begin{array}{c}\text { Hidden } \\
\text { Neurons }\end{array}$ & Layer 4 & Layer 3 & Layer 2 & Layer 1 \\
\hline 2 & 2.847 & 2.556 & 2.362 & 1.500 \\
\hline 3 & 2.711 & 2.476 & 1.722 & 1.547 \\
\hline 4 & 1.976 & 2.550 & 1.970 & 1.256 \\
\hline 5 & 2.464 & 2.701 & 1.704 & 1.235 \\
\hline 6 & 2.978 & 2.572 & 1.604 & 1.253 \\
\hline 7 & 2.256 & 2.618 & 1.624 & 1.165 \\
\hline 8 & 2.270 & 2.558 & 1.611 & 1.167 \\
\hline 9 & 2.368 & 2.561 & 1.682 & 1.346 \\
\hline 10 & 2.564 & 2.530 & 1.703 & 1.500 \\
\hline
\end{tabular}

\section{5) ANN retrieval using real satellite radiance data}

The generalization performances of the trained multilayer perceptrons were further evaluated with real satellite radiance data, from the High Resolution Radiation Sounder (HIRS-2) of NOAA-14 satellite. HIRS-2 is one of the three sonde instruments of the TIROS Operational Vertical Sounder (TOVS).

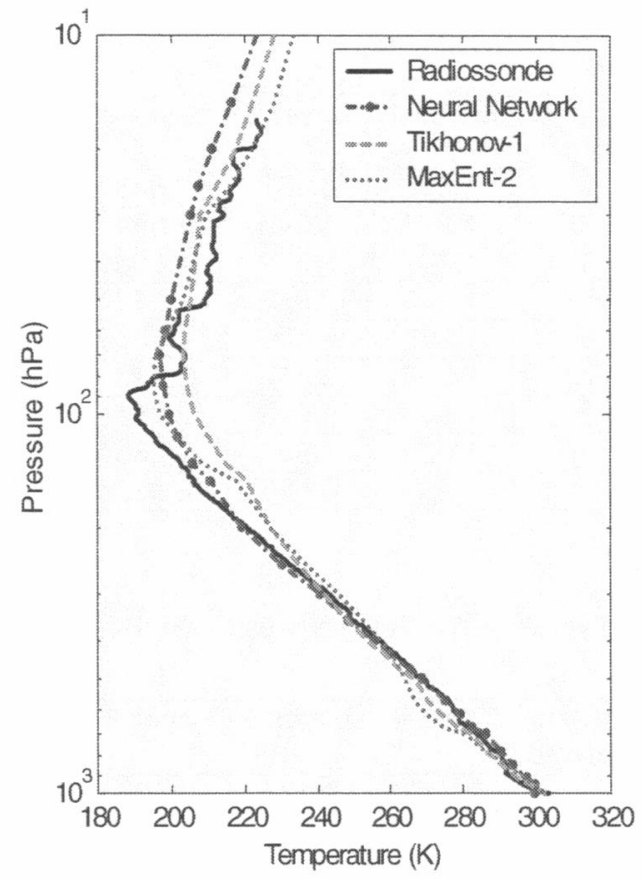

Figure 2. Retrieved temperature profiles using radiance data from NOAA-14 satellite (ANN trained with SDB1)

The results generated by the multilayer perceptrons were compared to in situ radiosonde measurements and the results obtained by [1] and [2], with Tikhonov and Maximum Entropy Principle of second order regularization techniques.

The number of observations is a fraction of the number of temperatures to be estimated. In the example discussed here, 28 temperature values are estimated from 7 radiance measurements.

In this case exemplar, the error analysis also consisted in analyzing the ANNs' performance trained with different databases. Tables 4, 5, and 6 show the errors associated to retrievals for ANN-MLP using different number of neurons in the hidden layer.

Figures 2, 3 and 4 show the results of the generalization tests. It is to be noticed there is a reasonable agreement between ANNs' retrievals and radiosonde measurements.

Table 4: Error for ANN using SDB1.

\begin{tabular}{|c|c|c|c|c|}
\hline $\begin{array}{c}\text { Hidden } \\
\text { Neurons }\end{array}$ & Layer 4 & Layer 3 & Layer 2 & Layer 1 \\
\hline 2 & 4.688 & 1.589 & 2.092 & 0.754 \\
\hline 3 & 3.900 & 1.589 & 2.695 & 0.769 \\
\hline 4 & 4.132 & 1.351 & 1.941 & 0.939 \\
\hline 5 & 3.441 & 1.453 & 1.669 & 0.745 \\
\hline 6 & 3.449 & 2.054 & 0.483 & 1.102 \\
\hline 7 & 3.853 & 1.249 & 1.223 & 0.577 \\
\hline 8 & 3.144 & 1.649 & 2.103 & 0.741 \\
\hline 9 & 2.773 & 2.113 & 1.606 & 0.629 \\
\hline 10 & 3.212 & 2.185 & 0.649 & 0.635 \\
\hline
\end{tabular}


Table 5: Error for ANN using TIGR.

\begin{tabular}{|c|c|c|c|c|}
\hline $\begin{array}{c}\text { Hidden } \\
\text { Neurons }\end{array}$ & Layer 4 & Layer 3 & Layer 2 & Layer 1 \\
\hline 2 & 1.003 & 3.467 & 1.848 & 1.693 \\
\hline 3 & 1.368 & 4.240 & 1.644 & 1.580 \\
\hline 4 & 1.243 & 3.879 & 1.680 & 1.424 \\
\hline 5 & 1.239 & 3.458 & 1.932 & 1.222 \\
\hline 6 & 1.230 & 3.499 & 2.135 & 1.428 \\
\hline 7 & 1.205 & 3.517 & 2.143 & 1.273 \\
\hline 8 & 1.586 & 4.093 & 2.241 & 1.368 \\
\hline 9 & 1.229 & 3.537 & 2.265 & 1.382 \\
\hline 10 & 1.231 & 3.636 & 2.337 & 1.432 \\
\hline
\end{tabular}

Table 6: Error for ANN using SDB1+TIGR.

\begin{tabular}{|c|c|c|c|c|}
\hline $\begin{array}{c}\text { Hidden } \\
\text { Neurons }\end{array}$ & Layer 4 & Layer 3 & Layer 2 & Layer 1 \\
\hline 2 & 2.719 & 3.174 & 3.597 & 1.031 \\
\hline 3 & 2.538 & 3.609 & 1.789 & 0.874 \\
\hline 4 & 1.491 & 4.909 & 2.007 & 0.677 \\
\hline 5 & 1.825 & 4.184 & 0.875 & 0.517 \\
\hline 6 & 2.900 & 2.949 & 1.537 & 0.846 \\
\hline 7 & 1.393 & 4.149 & 2.149 & 0.657 \\
\hline 8 & 1.675 & 3.768 & 2.635 & 0.762 \\
\hline 9 & 1.915 & 4.017 & 1.553 & 0.882 \\
\hline 10 & 2.266 & 3.777 & 1.220 & 1.305 \\
\hline
\end{tabular}

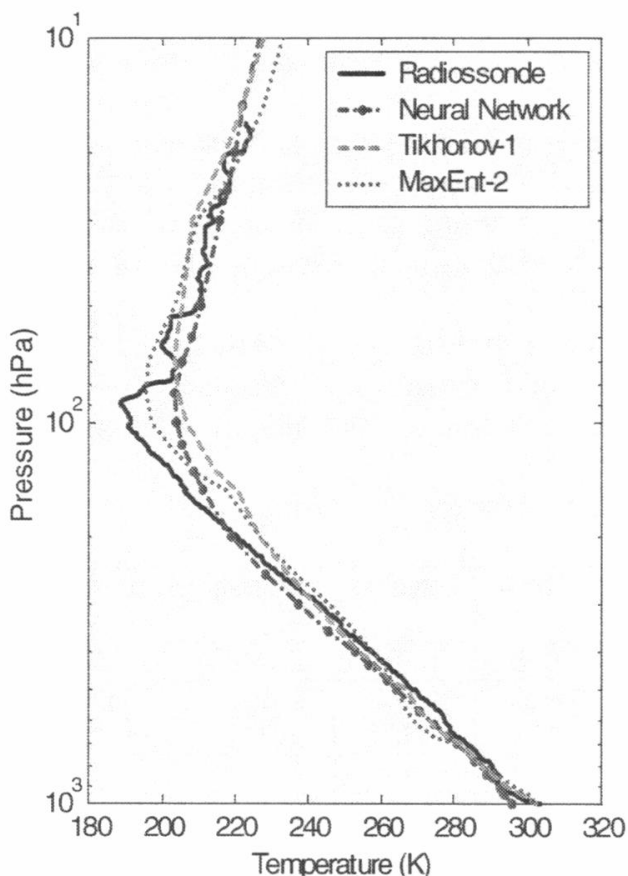

Figure 3. Retrieved temperature profiles using radiance from NOAA-14 satellite - result for TIGR database.
It should also be noticed that the ANN trained with SBD1 dataset presented the best performance. That is probably because the temperature profiles used during in the learning phase are closer to regional climate means. However, the combination of the SDB1 with the TIGR dataset improved the retrievals.

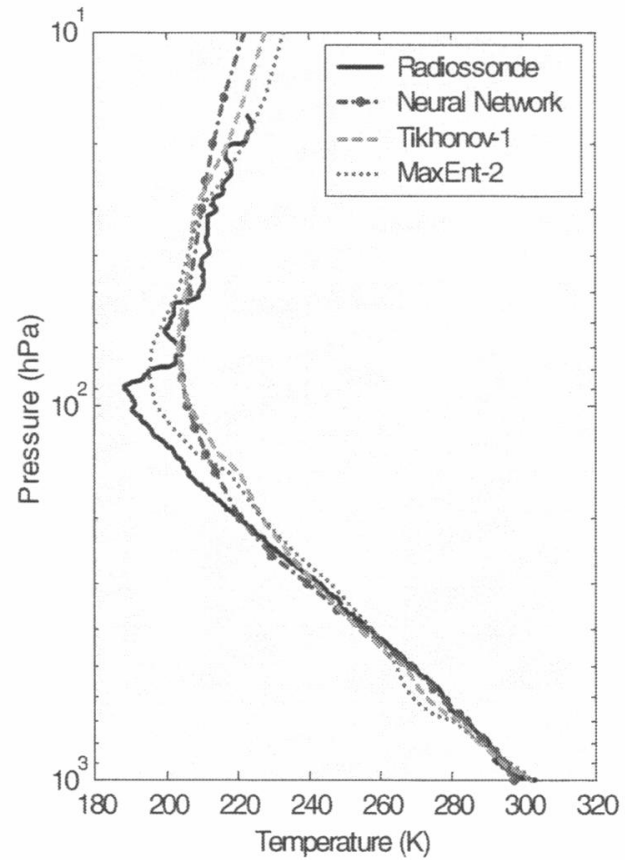

Figure 4. Retrieved temperature profiles using radiance data from NOAA-14 satellite - SDB1+TIGR dataset.

\section{6) Conclusions}

This paper presents an ANN approach for the problem of retrieving vertical temperature profiles of the atmosphere, which is a problem whose mathematical formulation is given by the integral radiative transfer equation, and leads to the solution of a highly illconditioned Fredholm integral equation of the first kind.

The results summarized in Tables 1, 2, 3, 4, 5, and 6 show the effectiveness of the multilayer perceptrons to solve this inverse problem, and the reconstructions are comparable with those obtained with regularization methods [1, 2], even for with noise contaminated data. However, the use of ANNs does not decrease the degree of the inherent ill-posedness of the inverse problem.

In general, operational inversion algorithms avoid being trapped in local minima by starting an iterative search process from an initial guess solution that is close enough to the true profile. Nevertheless, the dependence of the 
final solution upon a good choice of the initial guess represents a fundamental weakness of such algorithms, especially when less a priori information is available [12]. The use of ANNs may relax this constrain by increasing the training dataset.

It is important to notice that the ANN performed well in layers 1 and 2, which are the most important ones for meteorological applications.

The use of ANNs to solve the problem stated in this paper may imply in a search time reduction, thus making ANNs faster than regularization techniques. Also, the ANNs' features make them appropriate for hardware-based solutions that may be placed directly onboard satellites.

This paper presents preliminary results of an ongoing research that investigates inverse solutions for this problem that may lead to a release in the amount of processing power required to run climate or weather forecasting. New databases have been collected and the obtained neural networks will further be evaluated and compared with other ANNs approaches. Another preliminary result can be found with non-linear Hopfield's neural network in [13].

\section{References}

[1]. J.C. Carvalho, F.M. Ramos, N.J. Ferreira H.F. de Campos Velho, Retrieval of Vertical Temperature Profiles in the Atmosphere, 3rd International Conference on Inverse Problems in Engineering (3ICIPE), Proceedings in CDROM, under paper code HT02 (1999) - Proc. Book: pp. 235-238, Port Ludlow, Washington, USA, June 13-18, UEF-ASME (2000).

[2]. F.M. Ramos, H.F. de Campos Velho, J.C. Carvalho, N.J. Ferreira, Novel Approaches on Entropic Regularization, Inverse Problems, 15(5), 11391148,(1999).

[3]. C. D. Rodgers, Retrieval of the atmospheric temperature and composition from remote measurements of thermal radiation, Rev. Geophys. Space Phys., 14, 609-624 (1976).

[4]. S. Twomey, Introduction to the matehmatics of inversion in remote sensing and interative measurements, Amsterdam, Elsevier Scientific, (1977).

[5]. M. T. Chahine, Inverse Problem in Radiative Transfer: determination of atmospheric parameters, Jour. Atmos. Sci., 27, 960 (1970).

[6]. W. L. Smith, H. M. Woolf, A. J. Schriener, Simultaneous retrieval os surface and atmospheric parameters: a physical analytically direct approach, Adv. In Rem. Sens., 7 (1985).
[7]. K. N. Liou, An introduction to atmospheric radiation, academic press, Orlando, (1982).

[8]. S. Haykin, Neural Networks: A Comprehensive Foundation, Macmillan. New York, (1994).

[9]. C-T Lin and G. Lee, Neural Fuzzy Systems: A NeuroFuzzy Synergism to Intelligent Systems, Prentice Hall, New Jersey, (1996).

[10]. M. Nadler and E.P. Smith, Pattern Recognition Engineering, John Wiley \& Sons, New York, (1993).

[11]. L.H. Tsoukalas and R.E. Uhrig, Fuzzy and Neural Approaches in Engineering, John Wiley \& Sons, New York, (1997).

[12]. A. Chédin, N. A. Scott, C. Wahiche, P. Moulini, The improved initialization inversion method: A high resolution physical method for temperature retrievals from TIROS-N series. J. Climate Appl. Meteor, 24, (1985), 128-143.

[13]. V.C. de Viterbo, J.P. Braga, E.H. Shiguemiri, J.D.S. da Silva, H.F. de Campos Velho (2004): Atmospheric Temperature Retrieval using Non-linear Hopfield Neural Network, Inverse Problems, Design and Optimization Symposium (IPDO), 17-19 March, Rio de Janeiro (RJ), Brazil, Proceedings in CD-Rom, paper code IPDO-119.

[14]. P. Chandra. Sigmoidal Function Classes for Feedforward Artificial Neural Networks. Neural Processing Letters 18: 185-195, (2003).

[15]. Chevallier, F.; Cheruy, F.; Scott, N.A.; Chedin, A. A neural network approach for a fast and accurate computation of a longwave radiative budget. Journal of Applied Meteorology, 37 (11): 1385-1397, (1998)

[16]. A.N. Tikhonov and V.I. Arsenin, Solutions of Illposed Problems, John Wiley \& Sons, 1977.

[17]. NAG, E04UCF routine, NAG Fortran Library Mark 17, Oxford, UK (1995) 